collaboration between the physician who utilizes OMT and the respective clinical specialist. Perhaps this partnership may also be extended in the area of clinical research.

With all of the potential challenges and opportunties mentioned here, perhaps none is greater than devising a way to ensure that cardiac patients continue to receive OMT from their primary care physicians. This is likely to be enhanced if clinical studies reinforce the value of this modality. At the Detroit Osteopathic Hospital, 300 open heart surgical procedures are performed annually. Each of these patients undergoing these procedures routinely receives manipulative management postoperatively as part of a comprehensive treatment protocol. On hospital discharge, each patient is referred to an osteopathic physician for follow-up care.

However, we do not know how many patients continue to receive OMT. Thus, not only must we devise methods to gauge treatment follow-up, we must encourage primary care physicians to incorporate OMT in the overall medical management of such patients.

FELIX J. ROGERS, DO

Detroit Heart Institute

Detroit Osteopathic Hospital

Highland Park, Mich

MARY E. STARZINSKI, DO

St Clair Shores, Mich

Dr Rogers is a member of the JAOA Editorial Advisory Board.

\section{Will Medical Waste Tracking Act clean up environment?}

As medical debris washed up along the eastern seaboard during the summer of 1988 , state and federal legislators wasted no time proposing laws to control the problem. Much of this legislation, particularly the Medical Waste Tracking Act passed by Congress in June 1989, targets physicians as medical waste "generators."

The act authorizes a 2-year pilot program implemented and enforced by the Environmental Protection Agency (EPA) and individual states. The regulations require documentation of waste disposal. This entails tracking waste from its initial origin (physicians', dentists', and veterinarians' offices; clinics; hospitals; and nursing homes) through the hauling and disposal process.

Regulated waste categories include: cultures and stocks of infectious agents; human whole blood and blood products; human pathologic waste, including waste generated from surgery and autopsy procedures; contaminated animal carcasses from medical research; and sharp instruments, such as needles and scalpels.

Currently, Connecticut, New Jersey, New York, Puerto Rico, and Rhode Island are participating in the pilot program. The regulations may be instituted nationwide, depending on the outcome of this trial.

Under the Medical Waste Tracking Act, violators can be fined up to $\$ 25,000$ per day in civil penalties and $\$ 50,000$ per day in criminal penalties. Willful violators may be sentenced to a maximum of 5 years in prison.

The EPA estimates the program will cost physicians and small hospitals about $\$ 70$ and $\$ 3,750$ per year, respectively. However, actual costs may be 5 to 10 times greater.

Certainly, environmental pollution is cause for concern. However, the question arises whether the amount of medical waste generated warrants such imposing costs. Less than $10 \%$ of the debris that washed up on New York's shores during the summer of 1988 was medically related.

In light of this fact, the American Osteopathic Association has expressed concern that EPA action on this matter is taking place without sufficient information from the medical community. As a result, action was taken to have 
out sufficient information from the medical community. As a result, action was taken to have the AOA spearhead communications with the EPA to ensure that close communication is maintained with representatives of the medical industry.

What effect the Medical Waste Tracking Act will have on the environment remains to be seen. Meanwhile, other significant air and water pollutants continue to choke our natural resources.

\section{HHS Secretary Louis W. Sullivan to keynote AOA Convention}

Secretary of the US Department of Health and Human Services Louis W. Sullivan, MD, will deliver the keynote address at the 94th Annual AOA Convention and Scientific Seminar, Anaheim, Calif, November 12 through 16. Dr Sullivan, the first dean and president of the Morehouse School of Medicine in his native Atlanta, will focus on the healthcare problems of the underserved. The address will be given on Sunday, November 12, 1989, at 5:30 PM in the Anaheim Convention Center. 

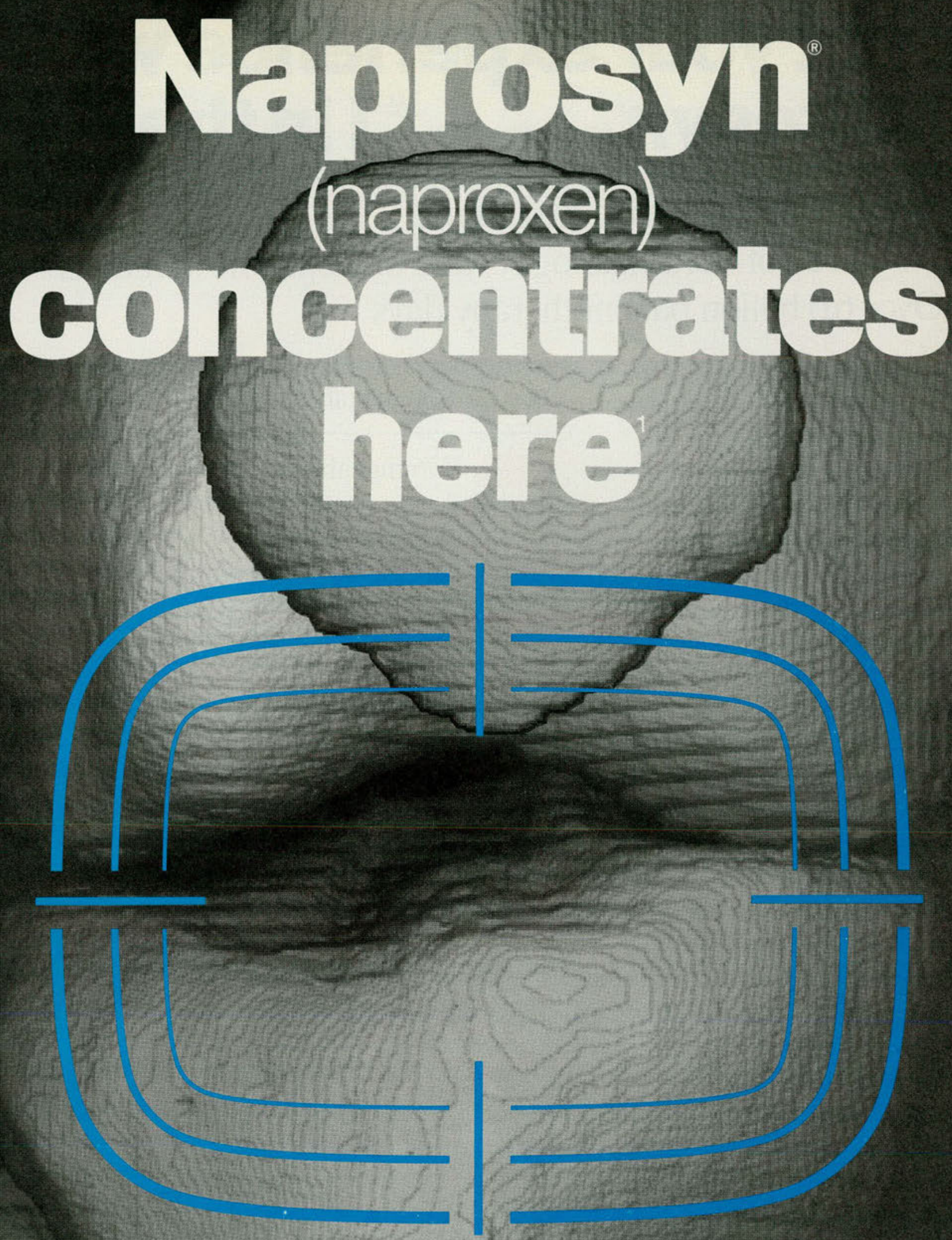

3-D CT image of knee.

Supplied by

California Advanced Imaging and Dimensional Medicine, Ine 


\section{Trust your experience with Naprosyn (naproxen): ${ }^{* 1}$ in the U.S.}

16 years of worldwide experience

\#1 choice of rheumatologists and orthopaedic surgeons** Over 6.6 billion patient therapy days

$250 \mathrm{mg}$ tablets

\section{Wide range of strengths and forms:}

$375 \mathrm{mg}$ tablets

$500 \mathrm{mg}$ tablets

Suspension $125 \mathrm{mg} / 5 \mathrm{~mL}$

(supplied in $16 \mathrm{oz}$ unit-of-use bottles)

\section{Concentrates in the joint-low risk to key organs'


-Leading industry audits for 12 months ending October 1988. Pharmacy sales of naproxen in the U.S. Data available upon request from Syntex Laboratories, Inc.

"Leading industry audit. A representative sample of 50 U.S. rheumatologists and 100 U.S. orthopaedic surgeons were asked which anti-arthritic they prescribe most often. In 1987 Naprosyn was named by $67 \%$ of rheumatologists and $33 \%$ of orthopaedic surgeons in this survey, which is conducted annually by an independ
firm. Data available upon request from Syntex Laboratories, Inc.

\section{Brief Summary}

Contraindications: Patients who have had allergic reactions to NAPROSYN, ANAPROX or ANAPROX DS or in tic reactions usually polyps, urticaria and hypotension associated with NSAID betore starting therapy If such symptoms discontinue the drug. Warnings: Serious Gl toxicity such as bleeding ulceration and perforation, can occur any time, with or without warning symptoms, in patients treated chronically with NSAIDs. Remain alert to ulceration and bleeding in such patients even in the absence of previous GI tract symptoms. In clinical trials, symptomatic upper Gl uicers, gross bleeding or perforation appear to occur in approximately $1 \%$ of patients treated for 3-6 months, and in about 2-4\% of patients treated for one year. Inform patients about the signs and/or symptoms of serious GI toxicity and what steps to take if they occur. Studies have not identified any subset of patients not at risk of developing peptic uiceration and bleeding. Except for a prior history of serious Gl events and other risk tactors known to be associated with peptic uicer disease, such as alcoholism smoking, etc.. no risk factors (e.g. age, sex) have been associated with increased risk. Elderly or debilitated events are in this population range) sulficient benefit should be anticipated to offset the potentiol increased risk of CI toxicity Precaution DO NOT GIVE NAPROSYN* - NAPROXEN CONCOMITANTIY WITH ANAPROX ANAPROX 0 DS (NAPROXEN SODIUM) SINCE THEY CIRCULATE IN PL ASMA AS THE NAPROXEN ANION ACU interstitial nephritis with hematuria, proteinuria, and nephrotic syndrome has been reported. Patients with impaired renal function, heart tailure, liver dysfunction, patients taking diuretics, and the elderly are at greater risk of overt renal decompensation. If this occurs, discontinue the drug. Use with caution and monitor serum creatinine and/or creatinine clearance in patients with significantly impaired renal function. Use caution in patients with baseline creatinine clearance less than $20 \mathrm{~mL}$ minute. Use the lowest effective dose in the elderl or in patients with chronic alcoholic liver disease or cirrhosis. With NSAIDS, borderline elevations of liver test's may occur in up to $15 \%$ of patients. They may progress, remain unchanged, or be transient with continued heratic Elections including or scor ond inal in contis led clical trials in iess than $1 \%$ of patients. Severe if systemic manif or eliminated during therapy do so slowly and observe patients closely for acyerse effects, including adren insulficiency and exacerbation of arthritis symptoms. Determine hemolobin values periodically for patients with initial values of 10 grams or less who receive long-term therapy Peripheral edema has been reported Therefore use with caution in patients with and anti-inflammatory activities may reduce fever and inflammation, diminishing their diagnostic value. Conduct ophthalmic studies if any change or disturbance in vision occurs. For patients with restricted sodium intake, note that the suspension contains $8 \mathrm{mg} / \mathrm{mL}$ of sodium. Information for Patients: Side effects of NSAlDs can cause discomfort and, rarely, there are more serious side effects, such as GI bleeding. which may result in hospitalization and even latar outcomes. Physicians may wish to discuss with patients the potentia risks and likely benefits of NSAID treatment, particularly when they are used for less serious conditions where
treatment without NSAIDs may be an acceptable alternative. Patients should use caution for activities
tThe most frequent complaints are gastrointestinal. See "Warnings" and "Precautions" sections of prescribing information.

1. Katona G, Burgos R, Ortega E: Pharmacokinetics of a single dose of naproxen in plasma and synovial fluid. Proceedings, IX European Congress of Rheumatology

Wiesbaden, September 1979. Excerpta Medica, 1980, pp 41-45.

c) 1989 Syntex Puerto Rico, Inc

requiring alertness if they experience drowsiness, dizziness, vertigo or depression during therapy Laboratory Tests: Because serious GI tract ulceration and bleeding can occur without warning symptoms, follow follow-up Drug Interactions: Use caution when giving concomitantly with coumarin-type anticoagulants hydantoin sulforam Druglaboratory Test Interactions: The drug may decrease platelet aggregation and prolon bleeding time or ncrease urinary values for $17-\mathrm{ketogenic}$ steroids. Temporarily stop therapy for 72 hours be bre doing time or function tests. The drug may interfere with urinary assays of 5 HIAA. Carcinogenesis: A 2-year rat study showed 0 evidence of carcy inenicity Pregnancy: Category B. Do not use dugne pregi A 2-year ress cluary needed. Avoid use during late pregnancy. Nursing Mothers: Avoid use in nursing mothers. Pediatric Use: Single doses of $2.5-5 \mathrm{mg} / \mathrm{kg}$. with total daily dose not exceeding $15 \mathrm{mg} / \mathrm{kg} /$ day, are safe in children over 2 years of age. Adverse Reactions: In a study, Gl reactions were more frequent and severe in rheumatoid arthritis patients on $1,500 \mathrm{mg}$ /day than in those on $750 \mathrm{mg} /$ day. In studies in children with juvenile arthritis, rash and prolonged bleeding times were more frequent, Gl and CNS reactions about the same, and other reactions less requeht than in adults. Incidence Greater Than $1 \%$, Probable Causal Relationship. G. The most frequen stomatitis CNS: headache" dizziness; drowsiness: lightheadedness, vertipo Dermatologic: itchin (pru itus)" skin eruptions" ecchymoses" sweating purpura Special Senses: tinnitus" hearing disturbances visual disturbances Cardiovascular: edema* dyspnea* palpitations, General: thirst Incidence less Than 1\%: Proba ble Causal Relationship. GI: abnormal Iver function tests, GI bleeding and or pertoration, her (1) hematuria, interstitial nephritis, nephrotic syndrome, renal disease. Hematologic: agranulocytosis, eosinoph lia, granulocytopenia, leukopenia, thrombocytopenia. CNS: depression, dream abnormalities, inability to concentrate, insomnia, malaise, myalgia and muscle weakness. Dermatologic: alopecia, photosensitive dermatitis, skin rashes. Special Senses: hearing impairment. Cardiovascular: congestive heart failure. Respiratory: eosinophilic pneumonitis, General: anaphylactoid reactions, menstrual disorders, pyrexia (chills an fever). Causal Relationship Unknown: Hematologic: aplastic anemia, hemolytic anemia. CNS: cognitive dysGI: non. Detic GI ugceration. hyperglycemia hypolycemia Overdosage: May have drowsiness, hearthurn indigestion nausea vomiting Empty stomach and use usual supportive measures In animals 05 , kg of activated levels of naproxen. Caution: Federal law prohibits dispensing without prescription. See package insert for full Prescribing Information. 


\section{The new NOF four-page holiday card announcement brochure is on its way to you! Watch for it.}

The brochure (shown here) features a selection of 12 new colorful cards in a variety of artistic styles. Pick one!

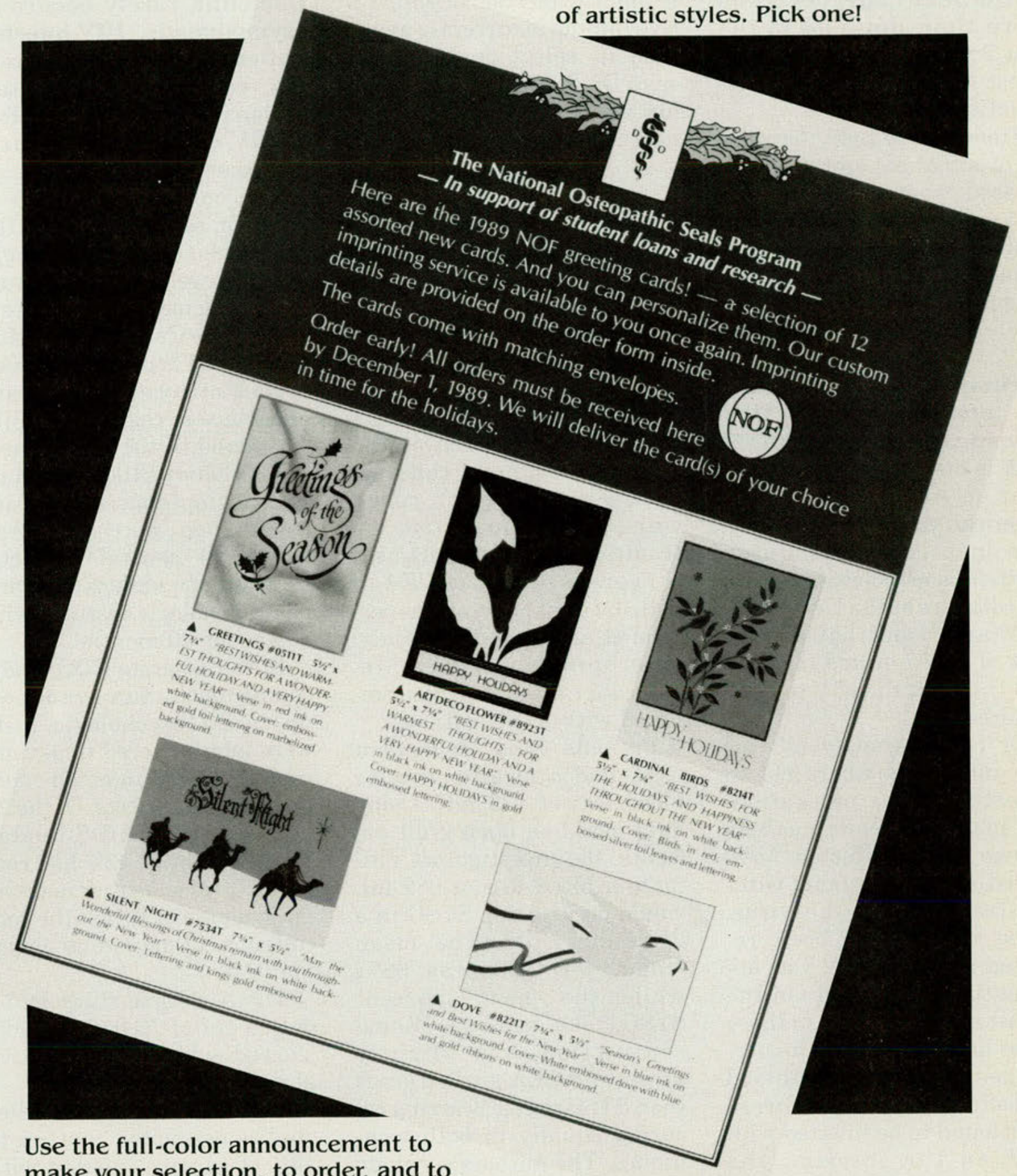
make your selection, to order, and to specify any custom imprinting you may want on your card and envelope.



Order Now.

Make certain you have NOF cards for the holidays. Use the announcement brochure to order. If you need another brochure, telephone us today at . . 312-280-5851 or 800-621-1773. 